\title{
LE JUGE DANS L'ETAT DE DROIT, LIBERAL ET DEMOCRATIQUE, GARDIEN DES LIBERTES ET DE LA DEMOCRATIE
}

DOI: $10.47743 /$ rdc-2015-1-0004

Slobodan MILACIC
Professeur émérite à l'Université Montesquieu-Bordeaux IV

\section{Résumé}

Le point de vue adopté dans ce "papier» vise à remobiliser la problématique du juge dans l'Etat de droit contemporain en le positionnant au sein du système démocratique et libéral, restructuré par le néolibéralisme. Le juge apparaît alors non seulement comme le "gardien des libertés» fondamentales, c'est-à-dire essentiellement individuelles, mais aussi comme le gardien des normes et de l'esprit des normes démocratiques, relatives aux institutions et procédures politiques, qui constitue l'autre profil du juge, relativement négligé par les discours ambiants. A ce double titre le travail du juge constitutionnel implique aussi et nécessairement le politique, qu'il n'y a pas lieux de dénoncer trop rapidement au motif de la "politisation du juge» et du danger d'un "gouvernement des juges»! D'autant plus qu'au-delà du droit et du juge, le système aujourd'hui désigné de façon raccourcie comme l' "Etat de droit» est structurellement garanti par les valeurs et les procédures plus spécifiquement démocratiques. Si le juge garantit la norme, c'est la démocratie pluraliste qui établit la norme libérale et garantit le juge, avec son statut de "troisième pouvoir»; primordialement juridique, mais avec des implications politiques-légitimes, puisque la démocratie, elle-même, est substantiellement politique ou elle n'est pas.

Mots-clés: démocratie; l'Etat de droit; juge constitutionnel; indépendance des juges; séparation et l'équilibre des pouvoirs

\section{Introduction}

Le néolibéralisme a induit et fini par installer une logique prévalant de fragmentation ${ }^{1}$ à tous les niveaux et dans tous les espaces du pouvoir et, par conséquent, du droit. Dans le domaine des idées, également, c'est la «spécialisation» des disciplines et des thèmes ${ }^{2}$ qui règne en maîtresse. Le fameux «néo-individualisme», dénoncé ou célébré, doit être compris

\footnotetext{
${ }^{1}$ La «mondialisation» ou «la globalisation», elle-même, implique la concurrence éclatée, au moins au départ..., et la localisation (voire la «délocalisation») comme réponse dialectique, pour ainsi dire inévitable.

2 «Questionnements», «problèmes», voire «problématiques» de plus en plus «pointus».
} 
de façon large, à la fois au sens propre et au sens figuré; en incluant des thématiques très «individualisées» c'est-à-dire spécialisées; d'une façon générale comme dans nos disciplines juridiques et politiques. Avec, cependant, en contre partie dialectique de ce discours "spécialiste», plus ou moins "étroit», un discours ambiant généraliste, voire idéologique, sur les «droits de l'homme». Mais entre le technique des discours disciplinaire et l'idéologique du discours idéologique ambiant on évite souvent le maillon politique, c'est-à-dire spécifiquement démocratique, pourtant indispensable; à la fois, à la bonne compréhension comme à la "bonne gouvernance» du système libéral-démocratique qui nous gouverne. Le paradigme juridique des droits de l'homme et du citoyen encadre désormais la problématique politique de la démocratie.

Le juge, en tant que protagoniste majeur des temps néolibéraux et «postmodernes» est trop souvent envisagé tel qu'en lui-même; en soi et pour soi, en questionnant les problèmes de son indépendance réelle, sa légitimité propre, ses pouvoirs croissants, sa jurisprudence et son efficacité finale. Mais il est rarement replacé, de façon globalisante, au sein du système de l'Etat de droit, qui est substantiellement, à la fois, libéral et démocratique, sur le plan des valeurs fondatrices et juridique et politique, sur le plan technologique, si l'on peut dire. Car, comme on le constatera plus longuement, si le libéralisme est "par nature», par vocation ou par simple constat, profondément juridique, avant d'être politique, la démocratie, elle, est essentiellement politique, avant d'être juridique. Malgré et contre quelques velléités doctrinales prétendant qu'elle est désormais avant tout "constitutionnelle», en marginalisant le politique-démocratique, considéré comme une conséquence du libéralisme bien compris! Or, la constitution est d'abord un acte politique, exprimant un compromis politique substantiel qui lui donne vie et la met «en vigueur» par un acte de volonté politique concrétisé par le vote. Sur la lancée de ce point de vue la démocratie devient le paradigme juridique et se présente sous forme d'Etat légal-traditionnel («de droit», quand-même...) ou de droit constitutionnel, qui monopolise aujourd'hui l'appellation "l'Etat de droit», en impliquant le contrôle de la constitutionnalité comme critère décisif. Le politique et le juridique étant envisagés ici comme des technologies organisatrices et régulatrices du système, c'est-à-dire des rapports globaux des sujets ou des acteurs au sein de l'Etat. Avec les techniques qui deviennent, à leur tour, des valeurs, en se confondant avec celles dont elles ne sont, au départ, que des instruments d'action. Comme, par exemple, le droit qui proclamée garantit les libertés individuelles. Le droit est donc indispensable au libéralisme, envisagé comme un système (ici «sous-système») sui generis, tout comme à la démocratie, stricto sensu, qui a besoin d'un "ordre» stable et transparent. Seulement, entre le libéral et le démocratique il ne s'agit pas strictement du même droit substantiel, ni du même ordre de fonctionnalité ou de préférences entre les procédés juridiques et politiques. C'est, précisément le croisement, c'est-à-dire, à la fois, la synergie et les contradictions des valeurs et des technologies invoquées qui font la complexité, voire la sophistication, c'est à dire le génie de notre système. Bien sûr, ceci oblige de rappeler que toutes les institutions, procédés et procédures que le mot technologie implique ne sont pas politiquement neutres ou idéologiquement aseptisés. Ils sont, en quelque sorte, les inventions et les instruments appelés ou induits par des valeurs 
fondatrices des systèmes. Ainsi, il faut le rappeler, aussi, ces technologies ont des utilités diverses, voir perverses, pour des systèmes différents que l'histoire moderne a connus. D'où la nécessité de recourir au droit substantiel comme critère déterminant. Car, la norme juridique et le vote politique ont pu être mis aux services des systèmes diamétralement opposés, par rapport à nos propres usages, démocratiques et libéraux. Des Etats et des systèmes "voyous» et "pervers» ne peuvent se passer techniquement du droit, comme structure d'ordre, ni idéologiquement de l'affichage de la liberté et de l'égalité, comme demande sociétale. A la fois par réalisme, pragmatisme ou opportunisme, c'est-à-dire par nécessité fonctionnelle et par souci de propagande légitimant!

Pour situer le juge constitutionnel au sein de notre système étatique, juridique et politique concernant les technologies institutionnelles, et libéral et démocratique sur le plan des valeurs et des principes, nous proposons d'esquisser d'abord le jeu complexe d'articulations de ces quatre variables, avant d'envisager le positionnement du juge, au carrefour de ces variables. L'espace juridictionnel s'avère complexe, à l'image du système luimême, entre les bipolarités des valeurs et celles des technologies, souvent complémentaire, mais souvent, aussi, concurrentes; parfois même carrément contradictoires. Notre système est, répétons-le, fondamentalement bipolaire, même là où on n'attend pas cette bipolarité: dans le consensus qui met une sourdine à l'opposition, mais pas forcément à la différence, alias concurrence. Car, le postulat pluraliste nous le rappelle: «Chassez la différence, elle revient au galop», dès que le consensus entre en action, c'est-à-dire en application. L'exécution est ici plus complexe, voire compliquée, que le constat du consensus. Et, c'est précisément à ce stade qu'intervient prioritairement le juge.

Comme tous les pouvoirs, acteurs et interprètes implicites de la constitution qu'ils appliquent, mais plus spécifiquement que les autres, le juge doit arbitrer entre les valeurs et les principes qui se trouvent en concurrence. Et de ce fait, aussi et nécessairement, entre les places respectives du juridique et du politique.

C'est pourquoi nous croyons utile de situer, au préalable et de façon diagonale, la structure profonde du système à travers sa longue formation historique, avant de nous intéresser au juge au sein de cette bipolarité sous sa forme actuelle, c'est-à-dire néolibérale.

\section{Sur l'Etat de droit bipolaire: libéral et démocratique}

Depuis le grand virage historique du néolibéralisme, impulsé par la crise pétrolière de 1973 et les conséquences par elle induites, notre système de gouvernement a substantiellement changé, "de la cave au grenier». Les valeurs libérales et individuelles sont propulsées comme désormais "fondamentales», en éclipsant d'autant les valeurs collectives, plus spécifiquement politiques, c'est-à-dire démocratiques, contrairement à une certaine vulgate sur le «patrimoine commun», concept plus souvent invoqué qu'analysé, comme «intouchable»; définitivement cristallisé et, en quelque sorte unanimitaire, ce qui n'est pas tout à fait le cas! Le paradigme juridique a suivi l'installation du néolibéralisme et 


\section{Slobodan MILACIC}

s'est imposé comme dominant, face au paradigme politique, qui, de surcroit, a eu à subir la nouvelle hégémonie de l'économique, comme aux origines, il y a deux ou trois siècles, selon la façon de calculer... Un large espace a ainsi était ouvert au Marché «libre» et ses «lois»économiques, transposées au politique; réduisant par là la place de l'Etat, c'est-à-dire du politique en général, atteint dans sa spécificité et son autonomie. En n'oubliant jamais que lorsque l'on parle du politique c'est le démocratique qui est concerné, substantiellement.

On est passé rapidement, sans débat d'idées correspondant à l'ampleur et à la gravité du changement, d'un système pour ainsi dire historique de la démocratie «libérale» ou "pluraliste», avec son "Etat légal», qui faisait une large place au "politique d'abord», au système actuel, avant tout libéral, avec son «Etat de droit» - constitutionnel, «saisissant» en bonne partie le politique par le droit et en en transférant une autre partie à l'espace privé de la société civile, à son économie et ses lois...

Ainsi, les deux composantes principales, libérale et démocratique, de notre système se retrouvent-elles dans un nouveau rapport hiérarchique ou le libéral domine le démocratique. Dans le sillage du néolibéralisme triomphant c'est le juridique qui s'affirmera, en induisant un déclassement du politique, en tant que référencie légitimant et, par conséquent, en tant que pouvoir arbitral. D'où, la montée en puissance du contentieux juridique, au détriment du contentieux politique, avec le juge constitutionnel comme symbole proéminent.

C'est sur la lancée de la promotion néolibérale des libertés, affirmées par le droit et garanties par le juge, que celui-ci finit par prendre une figure de Héros de temps postmodernes, gardien du temple constitutionnel des libertés les plus «fondamentales».

Le juge accapare ainsi un espace d'intérêt considérable: dans l'opinion publique, notamment, et aussi dans la doctrine juridique, naturellement. En tant que l'un des trois principaux pouvoirs de la Cité, cependant, il n'a pas le monopole de légitimité et, à ce titre, ne peut avoir de façon absolue le "pouvoir du dernier mot», comme par une certaine facilité et idéologie juridiste on le lui attribue parfois, en se contentant de la dogmatique et des apparences juridiques formelles. Personne, aucune instance ou aucun pouvoir institué dans une démocratie pluraliste ne peut avoir seul le pouvoir du dernier mot, car, "globalement et en dernière instance» celui-ci appartient au peuple, Souverain "en personne», s'exprimant directement sur le fond ou sur les conditions de procédure choisies comme légitimes pour les arbitrages décisifs «en dernière instance». D’ailleurs, au vu de la nouvelle montée en puissance et en popularité du juge, le problème de sa légitimité revient en discussion; notamment en France, dont la réserve historique face au pouvoir du juge est bien connue. Il est d'ailleurs resté une simple "autorité» de l'Etat dans la sémantique constitutionnelle «n vigueur ». La mouvance démocratique, pour sa part, lui cherche la légitimité en quelque sorte politique, c'est-à-dire électorale. En amont, c'est la question de son lien, direct ou indirect, avec le suffrage universel; en aval, c'est la question, toujours actuelle, d'un régime en adéquation avec sa nouvelle stature, ses nouvelles responsabilités fonctionnelles et réelles, au-delà du disciplinaire, déontologique, et pénal. La mouvance libérale se contente de sa légitimité juridique, c'est-à-dire constitutionnelle; elle trouve les critères de sa légitimation dans son statut constitutionnel et légal d'indépendance et son 
niveau de compétences, principalement. Le juge devient ainsi le «représentant du peuple» par le biais de la constitution, puisque il se prononce "au nom du peuple français». Les libéraux "purs et durs» estiment que cette "caution constitutionnelle» suffit, puisque la constitution exprime une certaine légitimité démocratique grâce au vote.

En ce qui concerne le «nouveau juge» ou le nouveau rôle du juge constitutionnel sous le néolibéralisme, force est de constater que certains paramètres de sa légitimité ont plus ou moins bougé; en droit ou en fait. Le souci de sa représentativité politique (en fait, mais en sourdine...) ou sociétale (plus facilement reconnue dans les systèmes anglo-saxons) devient plus réel et plus abouti. La légitimité constitutionnelle-fondatrice devient plus forte de signification: ainsi, les textes anciens sont souvent interprétés de façon assez radicalement renouvelée. Le renforcement de l'indépendance de la magistrature, en général, en droit ou en fait, ainsi que la remise à l'ordre du jour des divers types de responsabilités du juge contribuent au nouvel équilibre entre pouvoir et responsabilité, ce qui est une exigence typiquement démocratique. II n'empêche que, malgré tout cela, tout comme "une somme de villages ne fait pas une ville», des aspects éclatés d'une légitimité qui se cherche ne fait pas une légitimité globale cohérente, c'est-à-dire suffisante! En réalité, on retrouve en matière de justice le déséquilibre qui caractérise le nouvel Etat de droit, en général: le paradigme juridique-libéral est devenu écrasant par rapport au paradigme démocratique-politique. Le système, dans son ensemble, en a perdu l'équilibre nécessaire à son efficacité et sa légitimité, toujours interactives dans la durée, faut-il le rappeler.

En France, en particulier, la méfiance historique vis-à-vis du juge faisait partie de la culture traditionnelle, même sous la démocratie libérale passée, dont on gère encore les survivances aujourd'hui. On n'a pas voulu donner au juge un «supplément d'âme», c'est-à-dire de légitimité-démocratique, pour ne pas lui permettre d'acquérir plus de «pouvoir»! Par peur du politique on l'a écarté du démocratique. Dans beaucoup de pays les juges sont élus, même si leur élection acquiert un profil spécifique, du moins en fait, différent du suffrage politique tel qu'il fonctionne généralement. D'où, aux États-Unis, par exemple, une certaine représentativité des juges de la Cour Suprême; représentativité sociétale ou culturelle, mais d'une certaine façon géopolitique, voire politique au sens de sensibilité partisane. Les catégories ethniques (les noirs, les «latinos»), religieuses (les juges catholiques ou juifs) les genres (les femmes), les grandes régions y sont souvent représentées ès qualité. Sur le plan plus politique ou du moins idéologique, il est habituel d'y distinguer les «juges conservateurs» et les «juges progressistes», voire «pro-démocrates» et "pro- républicains». Le problème majeur en la matière n'y est pas perçu comme celui de leur connotation politique ou idéologique, mais comme celui de l'équilibre entre les «mouvances» représentées. Les juges étant des hommes, avec leurs idées, sentiments, voire passions et autres faiblesses dont ils ne peuvent se départir totalement et volontairement pour les besoins de la fonction, il est vain d'en espérer une neutralité désincarnée. Par ailleurs, la démocratie étant un système globalement rationnel, qui marie l'idéal avec les leçons de l'expérience, l'impossible ou l'improbable ne peut, en conséquence, être considéré comme souhaitable. A moins de sombrer dans l'utopie qui a fait couler beaucoup d'idéologies devenus systèmes "réels», 


\section{Slobodan MILACIC}

comme ce fut le cas avec le marxisme gouvernant. Prenant en considération ce paradigme réaliste on ne peut envisager légitimement, c'est à dire raisonnablement, que de prendre en compte, en la contrôlant, une certaine représentativité politique des juges du politique qui est la matière vivante du constitutionnel. A la fois une représentativité plus ouverte que politique au sens partisan, mais politique aussi, seulement «en flou», en quelque sorte; en transcendant les clivages partisans et en se situant dans les lignées des grands courants de sensibilités, des «familles politiques» plus ou moins informelles. En France c'est par les instances politiques de nomination, leur diversité et leur complémentarité que cet aspect des choses se réalise. Le reste étant laissé à la conscience et à la volonté des hommes pour "gérer» leurs «équations personnelles» conformément aux exigences déontologiques. ॥ n'empêche qu'aucun juge constitutionnel ne peut rester indifférent au «terrain»; au rapport de force du moment ou à l'état de l'opinion publique, avant de trouver la solution juridique.

L'exemple américain a été choisi ici comme le plus transparent, pour illustrer notre hypothèse sur les imbrications du juridique et du politique dans le champ de la justice constitutionnelle. Mais, d'une façon générale, dans le modèle de l'Etat de droit, libéral et démocratique, le juridique et le politique se côtoient et interfèrent nécessairement; seulement, à travers les combinatoires variables et mouvantes, selon les conjonctures historiques ou les rapports des légitimités systémiques, qui peuvent être, plutôt libérales et juridiques ou plutôt démocratiques et politiques. De ce fait, au lieu de craindre la "politisation du juge», son éventuel "gouvernement» illégitime, il vaut mieux admettre la part inévitable du travail politique qu'implique l'interprétation des normes, toutes, forcément à contenu politique, en contrôlant les divers équilibres dont les instances et les décisions sont sensés être des témoins. Car la vie démocratique ne s'arrête pas avec une sentence juridictionnelle. La ronde des pouvoirs et les ressourcements des légitimités sont continus et perméables, même s'ils connaissent des temps forts, des rythmes de croisière et des blocages.

\section{A. La construction historique de la bipolarité démocratique et libérale, en rappel}

Au début de la modernité postrévolutionnaire (1789) ce fut avant tout le libéralisme juridique, agrémenté progressivement de démocratie politique, au cours du XIX ${ }^{\circ}$ siècle, avec la montée en puissance du suffrage universel et des partis politiques. Le «libéralisme classique», comme système ouvert par excellence, avait précisément dégagé la voie à la démocratie, jusqu'à la prépondérance, voire la domination de celle-ci pendant un bon siècle, entre les années 1875-1877 et les années 1973-1975, en France. A partir de cette période le "néolibéralisme» marque un retour aux sources, plus ou moins revues et corrigées, en dominant désormais le politique - démocratique: à la fois sur le plan technologique du mode de fonctionnement, plutôt juridique, et sur le plan idéologique des valeurs, plutôt individuelles. La liberté et la norme juridique se trouvent fortement valorisées, par rapport au vote et la majorité - politiques, plus ou moins «déclassés» dans la grille des hiérarchies légitimâtes. 
A la sortie du siècle des Lumières, après l'acquis du libéralisme économique, ce fut dans la suite logique du développement du système, avec la Révolution Française de 1789, avec la mise en place du libéralisme politique; garant politique de l'ordre des libertés "fondamentales» de l'homme et du citoyen, parmi lesquelles les libertés économiques n'étaient pas les moindres. En tant que personnes ou individus, d'abord et, surtout, en matière de la propriété - "sacrée», et de la libre concurrence - régulatrice, sacrée ou du moins consacrée, elle aussi, comme principe de gouvernement. La liberté d'expression viendra donc à la suite et ne s'exercera qu'individuellement. Tout cela, l'économique et le politique, substantiellement pensés et normés sur une base individuelle, intervient avant le collectif, c'est-à-dire la démocratie politique. Celle-ci viendra s'y articuler progressivement, au cours du XIXème siècle. Grâce au suffrage universel et les partis politiques, qui se "consolident» pendant la seconde moitié du siècle, pour se stabiliser historiquement, non sans mal, sous forme de la république ou de la "démocratie parlementaire», à partir de la "constitution Grévy», en 1877. Avec la promotion des procédures et des valeurs plus spécifiquement démocratiques, comme la délibération «publique», alias transparente, le vote "universel et secret» et le compromis légitime comme exigence substantielle, les piliers fondateurs du système ont été mis en place. Mais, c'est surtout avec l'établissement d'un certain équilibre - concrètement suffisant - entre les deux volets des valeurs, libérales et démocratiques, sous -tendus par leurs technologies préférentielles, juridiques et politiques, que le système trouvera sa légitimité et son efficacité; ce qui garantira sa stabilité. Bien sûr, en matière des affaires humaine et sociétales l'équilibre ne peut-être que relatif et les effets pervers du déséquilibre ne se font sentir qu'à partir d'un certain «seuil d'intolérance» ou de perversion des usages.

Si le $X I X{ }^{\text {ème }}$ siècle a été "plutôt libéral», le $X X^{\text {ème }}$ siècle sera "plutôt démocratique». Après la lère guerre mondiale et - surtout - la crise économique de 1929, qui a débouché sur la demande de "plus d'État», c'est-à-dire d'un certain interventionnisme, voire dirigisme politique (en matière économique et sociale) et sa logistique institutionnelle de l'Etat pour réguler le marché défaillant. Cette mutation du rapport, en quelque sorte une inversion hiérarchique, entre le politique et l'économique, le public et le privé, était légitimée par les procédés d'action relevant de la démocratie politique qui y ressource sa légitimité pour le siècle à venir. Ce sont, en effet, la légitimité ressourcée du politique et les moyens d'action démocratiques qui en ont résulté, qui ont fini par donner à l'économique un nouveau statut juridique, désormais soumis à un certain encadrement politique («souple», "semi-souple» ou "semi-rigide», comme la planification). Avec une nouvelle «décote» idéologique de l'économique ${ }^{3}$ au profit des valeurs politiques ou, plus largement, économiques et sociales;

\footnotetext{
${ }^{3}$ On était loin, alors, du productivisme et du consumérisme effrénés, typiques de notre postmodernité, économiste et mondialisée. La fonction sociale de l'économie dont s'est saisi le discours politique, valorisait la distribution autant que la production. La démocratie politique était alors abondamment dans son inspiration égalitaire; autant «distributionniste» que «productiviste», accordant une grande importance à l'utilité sociale, «générale» ou publique, à côté de l'utilité économique, aujourd'hui déterminante ou, au moins, surdéterminante.
} 


\section{Slobodan MILACIC}

mais transitant toujours par les procédures et les discours d'idées - politiques; même si la finalité substantielle reste d'ordre plus spécifiquement économique et social.

Ainsi était affirmée la primauté des choix politiques sur l'économique et sur le juridique, qui suivront ${ }^{4}$ la filière politique et démocratique - dominante par la suite, au cours du $X X^{\circ}$ siècle, grosso modo.

De ce point de vue et de façon très générale, si le $X X^{\text {ème }}$ siècle a été celui de l'affirmation des «libertés publiques» et du libéralisme dominant, d'une façon plus générale, le $X X^{\text {ème }}$ siècle est celui de l'affirmation de la démocratie et du «politique d'abord». II s'agit, bien sûr, de la démocratie libérale, devenue "pluraliste», avec les valeurs collectives/ pluralistes et les techniques/procédures politiques souvent décisives; "souveraines», chaque fois qu'il il fallait faire appel à la «dernière instance». Ainsi, la place décisive revenait à la négociation, au compromis et au vote, qui se superposent à la triade typiquement libérale, fondée sur l'affirmation des règles, leur exégèse par les interprètes juridictionnels et la sanction du juge.

Le temps fort de la politisation, jumelée avec la démocratisation du système, constaté dans les «années 30», se répète après la llème guerre mondiale et les «années 50 ». L'économique a même été largement soumis au politique, avec les planifications «semirigides»... 5 . Le marché était ainsi en bonne partie encadré et contrôlé. Le pouvoir de contrôle du juge de droit commun s'arrêtait au niveau législatif, avec, à l'époque, simplement un «Etat légal», le politique régnant en maître, globalement, et surtout au sommet de la pyramide du pouvoir dans l'Etat. La «classe parlementaire» et "partisane» ou, plus exactement, "politique» était en quelque sorte souveraine; à la fois juge et partie, dans les faits, les croyances, sinon dans la doctrine juridique et le droit positif, parfois en décalage des usages. Les efforts, marginaux et plutôt symboliques, du contrôle de constitutionnalité sont restés sans réelle pertinence, jusqu'à la Vème république et les années $70-80$. C'est alors, précisément, que le droit commencera par "saisir le politique», en donnant de la crédibilité au nouvel «État de droit» - constitutionnel. Cela sera l'œuvre du virage néolibéral, juridisme et constitutionnaliste, de la décade 1970-1980. Mais, en attendant, entre la fin du XIXème siècle et la fin du XX ${ }^{\text {ème }}$ siècle, il s'agira du siècle démocratique, au point où certains systèmes, pourtant fondamentalement mixtes ou bipolaires, étaient désignés à partir de leur seul paradigme politique et démocratique: «le socialisme démocratique scandinave», "la démocratie chrétienne italienne», "la démocratie française» ou, simplement, "la démocratie parlementaire» ou «républicaine». Ceci même dans les manuels de Droit constitutionnel, sensés pourtant mettre en valeur surtout le paradigme juridique. Mais les auteurs, victimes de la mode ambiante des appellations scientifiquement pas très "contrôlées», se contentaient du politique, au nom de la démocratie et oubliant le

\footnotetext{
${ }^{4}$ On se souvient, cependant, du mal que le très populaire Président Roosevelt a eu pour imposer cette prévalence du choix politique à la société économique américaine (de culture libérale, s'il en fut) et, en droit, à la Cour Suprême. C'était vraiment un new deal!

${ }^{5}$ Selon le vocabulaire conceptuel très usuel de l'époque.
}

REVISTA DE DREPT CONSTITUŢIONAL 
libéral, «allant de soi», à l'époque; comme la démocratie sous le néolibéralisme, de nos jours!

C'est donc la démocratie, «libérale», "pluraliste» ou "constitutionnelle»; "scandinave», "anglo-saxonne» ou "latine» qui était une référence paradigmatique; pas le libéralisme "démocratique», ou "pluraliste», plus ou moins épuré et globalisant, comme dans notre contexte néolibéral du début $d u X X I^{\circ}$ siècle. Le qualificatif libéral n'apparaissait pas alors dans l'appellation générale du système, tout comme aujourd'hui le qualificatif démocratique n'apparait plus, sinon marginalement, dans l'appellation générique et usuelle de l'Etat de droit.

Le néolibéralisme apparaît dans un nouveau contexte, survenu pendant le siècle finissant et témoignant d'une certaine "usure du politique», voire un certain "désenchantement démocratique». La crise pétrolière des années 73-74, prélude à la crise économique quasiment continue depuis, provoque un profond bouleversement de notre échelle des valeurs référentielles et de nos technologies préférentielles. C'est le néolibéralisme dans le système de gouvernement étatique, mettant l'économique et son alter égo, le Marché, devant l'Etat politique. Valorisant ainsi l'espace privé, civil ou social, par rapport à l'espace public politique. Mettant l'individuel devant le collectif, vidant la délibération démocratique de son sens profond qui vise le compromis, et privilégiant l'exégèse et la transparence des règles en vigueur, comme critères de légitimité. On postule, désormais, que le choix rationnel et individuel est en quelque sorte préétabli, et désigne le fondement de «la bonne gouvernance» néolibérale que le consensus (de préférence) doit seulement «positivement» concrétiser. La perspective s'annonce comme libérée de l'exigence de l'alternative. On dévalorise ainsi l'idée démocratique du choix, par postulat obligatoire et plus ou moins équilibré et, jusque-là, le critère essentiel de la "bonne gouvernance » démocratique, cette fois-ci. Sur cette lancée, on neutralise le véritable débat pluraliste, plus conflictuel que concurrentiel, car la concurrence était déjà «codifiée» dans la science comme un concept qui est propre au marché et, comme tel, déjà pris en quelque sorte!

Le scénario historique s'est déroulé en cette fin de siècle de façon dissymétrique, par rapport à 1929. Au lieu de vouloir remédier à la situation de "pas assez d'État», c'est-à-dire de pouvoir politique (toujours identifié par le démocratique), c'est le «trop d'État» qui est dénoncé et tenu pour responsable de la crise, cette fois-ci. Il fallait donc «libérer le Marché» du "carcan étatique» pour lui permettre de retrouver son "autorégulation» et favorisant ainsi «la sortie de crise». Seulement, ce qui était présenté, à l'époque, comme un «miracle libéral ", c'était la solution de "sortie de crise» financière et économique, et non pas un changement profond et durable de notre système de gouvernement. Dans un premier temps c'est seulement de gestion, alias "gouvernance» qu'il était question. Il s'agissait des politiques néolibérales de Mme M. Tatcher et de M.R. Reagan et non pas du changement, en profondeur, des rapports généraux des composantes libérales et démocratiques de notre système. L'explosion, ou presque, du néolibéralisme comme un nouveau système de gouvernement s'est avéré être génératrice du nouveau déséquilibre structurel entre les deux paradigmes fondateurs. Cette «transition» là n'a pas été débattue comme telle. Ce sont les divers pouvoirs qui ont dû, au fur et à mesure, l'assumer, plus ou moins 


\section{Slobodan MILACIC}

progressivement. La place du juge dans ce processus pragmatique et continu a été énorme. C'est lui, l'interprète désigné du sens et de la portée, subtils et variables, des anciennes normes - reconduite par adaptation, et des normes nouvelles, à insérer dans la cohérence néolibérale.

Avec la primordialité des libertés individuelles - «naturelles» sur les choix collectifs politiques, c'est tout naturellement le paradigme juridique qui revient au premier plan. II accompagne, cette fois-ci, non seulement le principe de la légalité, mais celui de la constitutionnalité, sensé soumettre tout le politique (législatif et exécutif) au contrôle du juridique, grâce au juge constitutionnel aux compétences surclassées et à la légitimité renforcée. Ainsi est né le nouvel État de droit - constitutionnel, à la différence et en progression par rapport au Reichstadt allemand du XIXème siècle. C'est à partir des années 70-80 que le juge constitutionnel fut promu et remobilisé en Europe occidentale, terre de la démocratie libérale, comme la France, par exemple ${ }^{6}$. Auparavant, il est vrai, il y avait l'exception autrichienne, due à Kelsen, dans les années 20; on sait ce qu'il en est advenu dans les années trente. Et, surtout, il y avait les cas des ex-vaincus de la llème guerre mondiale. L'Allemagne et l'Italie étaient dotées de cours constitutionnelles comme garanties du respect des nouvelles constitutions libérales et démocratiques. Avec la structure fédérale (en Allemagne) ou régionale (beaucoup plus légèrement en Italie) des Etats, et, avec la promotion constitutionnelle des libertés publiques et même certaines normes internationales promues au rang des normes constitutionnelles, voire supra-constitutionnelles... Les nouveaux régimes étaient soutenus par le "parlementarisme rationnalisé», revu et corrigé une nouvelle fois et garanti par un juge constitutionnel en qui étaient placés les plus grands espoirs! II y avait aussi la logistique du Droit international, appelé à la rescousse. Le constitutionnalisme, mouvance essentiellement juridique, voit ainsi ses premiers fondements, avant que sa construction ne se généralise et se consolide avec le néolibéralisme, dans les années 80; entrainant dans son sillage les pays postcommunistes d'Europe Centrale et Orientale.

Entre la crise pétrolière des années 1973-1974, faisant tâche d'huile, et les "Évènements de l'Est» des années 1989-1990, toutes les stratégies des acteurs et les analyses des auteurs s'étaient placées sous la bannière du Conseil de l'Europe et de sa fameuse Commission de Venise, dont la devise était: "La démocratie par le droit». L'ambiguïté qui en résultera sera due à une certaine confusion conceptuelle entre la liberté et la démocratie, considérée comme synonymes par "constat» ou simple affirmation, qui se perpétue de nos jours, de façon assez générale, et dont la présente approche tend à rendre compte.

Avec le virage néolibéral, le souci de liberté, en tant que valeur référentielle, submerge le souci de démocratie. En tout cas, le libéralisme et ce que l'on pourrait appeler, par goût

\footnotetext{
${ }^{6}$ Ce n'est qu'entre 1971, 1974 et 2008, comme déjà évoqué, qu'il devient légitime de parler, pour la France, du «juge» constitutionnel, malgré la sémantique retardataire, qui évoque, elle, le «conseil», au lieu de cour, les «membres», à la place des juges et des «décisions», à la place des arrêts ou des jugements. Le tout dans le cadre constitutionnel général, qui, lui, évoque «l'autorité judiciaire» là où toutes les constitutions similaires affirment le «pouvoir judiciaire».
}

REVISTA DE DREPT CONSTITUŢIONAL 
de symétrie, le «démocratisme», sont considérés comme un couple fusionnel: inséparables dans la réalité et de ce fait inutilement séparées dans l'analyse, dit-on! Or, précisément, ils apparaissent séparément, de façon parfaitement différenciée, bien qu'en convergence, à travers l'histoire et nous paraissent, aujourd'hui, utilement séparables pour les besoins analytiques et critiques, car la domination, pour certains l'hégémonie néolibérale, perturbe profondément un système dont la qualité, pour ne pas dire la durabilité, dépend précisément et fondamentalement de l'équilibre entre ses deux paradigmes constitutifs évoqués. Pour revenir à la métaphore du couple fusionnel, on comprend aisément que la stabilité de la relation dépend de l'équilibre structurel de ses deux composants. L'hégémonie démocratique, sans le contrepoids libéral suffisant, peut induire "la tyrannie» ou le "despotisme de la majorité», qui débouche souvent sur celle d'une oligarchie politique, puis, celle d'un chef, leader plus ou moins charismatique", dont le "culte» se substitue à la majorité, c'est-à-dire au peuple...tout en développant un discours populiste. De son côté, le libéralisme insuffisamment encadré par le politique, c'est-à-dire par l'Etat démocratique, est alors, dans son idée abouti ou de freinée, gouverné par la loi de la "concurrence débridée», c'est-à-dire la «loi du plus fort», qui ignore l'exigence égalitaire, fondement de la démocratie. C'est pourquoi un certain vocabulaire parle du marché ou de la concurrence - «sauvages», propre à l'état de nature et contraire à toute civilisation. Car, finalement, l'histoire nous l'a démontré, le Marché n'est pas un véritable système (un modèle de système), dans la mesure où il ne s'autorégule pas; il a besoin d'une autorité extérieure et d'une légitimité supérieure, à savoir l'Etat politique. II n'y a pas de "main invisible» d'Adam Smith qui devait garantir l'auto-régulation du marché! Mais, il est vrai, le libéralisme n'est pas l'anarchisme, c'est-à-dire un libertinisme quelconque. Comme souvent rappelé par l'histoire économique, le libéralisme reconnait avoir besoin de l'Etat politique, mais un Etat minimaliste ou «modéré», c'est-à-dire "raisonnable», sans plus, pour reprendre le vocabulaire de la rationalité libérale. Plus concrètement, on le constate dans et par les faits: le capitalisme moderne ou postmoderne, c'est-à-dire l'économie libérale n'a pas un besoin vital de la démocratie ${ }^{8}$, alors que c'est l'inverse pour la démocratie politique. La relation est asymétrique. La démocratie libérale, elle, s'articule nécessairement avec l'économie libérale, pour des raisons multiples, d'ordre structurel ou systémique, qualitatif et quantitatif (niveau de développement suffisant et économie largement libérale) qu'il n'y a pas lieu de développer ici. Qu'il suffise de le constater.

La survenue du néolibéralisme au cours des années 80 a donc privilégié l'économique et surtout, pour ce qui nous intéresse, le juridique, garant des normes libérales sur la

\footnotetext{
${ }^{7}$ L'exemple le plus saillant que l'histoire nous ait fourni est celui du système soviétique avec le parti totalitaire, son politburo de la «direction collégiale» et son chef, «le petit père du peuple», objet du «culte de la personnalité». La France en a fait une courte expérience sous la Convention, avec la dictature jacobine, son Comité du Salut Public, notamment, et Robespierre au sommet de la pyramide en pointe...

${ }^{8}$ Des dictatures latino-américaines et asiatiques ont pu connaître un fort développement du capitalisme, car l'ordre public, la propriété privée et la concurrence y étaient assurés par des régimes autoritaires... L'exemple du Chili de Pinochet en est l'illustration la plus drastique. Sans s'attarder sur le cas de la Chine, qui, tout en étant plus complexe, relève de ce grand écart, en voie de stabilisation (?), entre l'économique, largement privatisé et concurrentiel et le politique, encore totalitaire ou, du moins, autoritaire.
} 


\section{Slobodan MILACIC}

propriété privée, la libre concurrence et les libertés des individus en tant que tels. Les citoyens, en politique, en sont le «complément» indispensable, dans la logique globale du système.

Pour le reste, le fonctionnement «ordinaire» ou «normal» du système relève principalement de la régulation normative, qui en est la technique privilégiée. La liberté individuelle est donc posée par-dessus le souci de son usage collectif, c'est-à-dire politique. La démocratie politique, avec sa valeur égalitaire comme fondement idéologique, conserve sa place, bien entendu; mais elle est désormais éclipsée par les éclats du néolibéralisme individualiste et juriste - triomphant.

\section{B. Le juge en tant qu'interprète des "valeurs partagées»}

La fonction d'interprète du juge implique un travail politique d'interprétation des valeurs consensuelles mises en cause, et du rapport contextualisé entre ces valeurs. Comme déjà évoqué, il doit articuler la structure du système et la conjoncture politique, l'esprit des lois et l'esprit du contexte. Car, nos "valeurs partagées» ne sont pas un bloc compact, à l'image du "bloc de constitutionnalité», souvent invoqué! Si les référentiels sont communs et un certain «noyau dur», très rapidement des nuances, différences, concurrences et parfois même oppositions s'affirment. Le juge est souvent, bien qu'implicitement, conduit à arbitrer entre ces valeurs; non seulement éventuellement perçues de façons contradictoire, mais aussi dans un rapport de légitimités respectives variable. C'est dire l'importance du juge en la matière, alors qu'elle n'est pas suffisamment mise en valeur.

L'arbitrage, explicite ou implicite, entre le libéral et le démocratique.

Il s'agit ici des "valeurs fondatrices» ${ }^{9}$, celles que l'on fait fusionner par la formule du "patrimoine commun des valeurs», alias "valeurs communes» ou "partagées», sans jamais cependant en approfondir l'analyse du contenu et les diverses connotations de la sémantique conceptuelle qui varie selon les époque, les acteurs et les cas de figures.

Cette analyse, critique par vocation, fait assez rapidement apparaître les différences, sources de divergences qui apaisent surtout dans les applications concrètes et particulières de ces valeurs. Que de lois «liberticides» parce que "égalitaristes» ou, symétriquement, «inégalitaires» parce que conduisant au «libéralisme sauvage». L'énoncé générique de la valeur, et du principe qui en est issu, peut faire croire au lien fusionnel entre la liberté et l'égalité, mais dès que l'on commence à préciser le contenu précis et ses implications, des différences, voire des contradictions, apaisent.

On oublie volontiers, de nos jours très consensualistes, que le fameux consensus, devenu le référentiel primordial de légitimation (plus fort que la majorité!) ne signifie pas, pour autant, l'unanimité, mais implique malgré ses apparences formelles un contenu compromissoire. Tout comme la majorité, mais de façon beaucoup plus transparente, dans ce dernier cas. D’une certaine manière, les consensus sont des compromis, seulement

\footnotetext{
${ }^{9}$ Les «droits fondamentaux» ne sont que le reflet des valeurs «fondamentales», dont ils sont inspirés et qui transitent par des principes constitutionnels, devenus plus nombreux et plus contraignants, comme les travaux du dernier Congrès mondial de droit constitutionnel de Mexico, en décembre 2010 l'ont révélé.
}

REVISTA DE DREPT CONSTITUŢIONAL 
"constatés», mais pas votés, ni délibérés dans la perspective d'un vote où l'on va pouvoir se compter! II s'agit d'un procédé sans vote, précisément et par définition, puisqu'il a été inventé justement pour éviter le vote «diviseur», révélateur des dissensus qui couvent toujours sous la braise pluraliste... et que par intérêt commun des principaux acteurs on tient à taire De surcroît, le consensus est obtenu sans négociation explicite, de type franc et direct, où, après que chacun ait exposé ses objectifs on condense les oppositions de façon bipolaire pour obtenir la bipolarité démocratique avec la majorité et l'opposition. Ceci étant constaté, la tendance à la normalisation des consensus politiques, au-delà du systémique, s'est accentuée avec le néolibéralisme. Aux débuts de la démocratie libérale le consensus était seulement systémique. II en va ainsi des fameux "consensus fondateurs», comme dans le cas des «vieilles» démocraties occidentales. Avec l'extension du procédé consensuel au domaine politique des précautions d'usages sont prises, précisément «pour ne pas réveiller le loup qui dort»; pour mettre en sourdine ou masquer la différence ou la conflictualité latente, sans quoi la fonction du procédé consensuel ne pourrait plus être assurée. Le consensus implique en ce sens un accord subtil, plus ou moins implicite, sur le désaccord mineur, pour sauvegarder un accord flou sur l'essentiel. Mais avec l'évolution historique et les variations conjoncturelles, le rapport entre les deux paramètres peut s'inverser et, de toute façon, le secondaire peut peser plus ou moins lourdement dans l'opérationnalité des choses, en nécessitant des adaptations du consensus initial aux contextes nouveaux! C'est l'ambiguïté profonde et essentielle du consensus qui induit toujours la nécessité des interprétations, comme ici celles des valeurs fondatrices par le juge, qui est appelé à faire ce travail en permanence, sinon au cas par cas. Or, il s'agit-là d'un travail autant juridique que politique, puisqu'il s'agit de faire rentrer dans les moules juridiques des réalités politiques différentes et fuyantes.

\section{Sur l'encadrement du juge par le système: entre le juridique et le politique}

L'éclairage du juge par le cadre bipolaire et fondateur permet de mieux le situer en tant qu'arbitre entre les valeurs, lesquelles, bien que consensuelles, sont parfois concurrentes et parfois même contradictoires, quant à leurs «sens et portées» concrètes. De plus, qui dit valeurs dit moyens de leur mise en œuvre et implique le choix des procédés ou des procédures juridiques ou politiques qui en sont plus ou moins induits. Ainsi, même lorsque le politique emprunte au juridique, il préfère une norme, une loi «républicaine» par vocation, car votée directement par la majorité politique, essentiellement égalitaire en tant que disposition "générale et impersonnelle», délibérée collectivement, dans la transparence de surcroît. Tandis que des «règles» concrètes, elles, sont apparamment moins liées aux exigences de la légitimité politique, alias démocratique et plus expressives de l'Etat "administratif», plus ou moins performant, ou de la gouvernance «technocratique». 


\section{Slobodan MILACIC}

\section{A. Le juge arbitre entre les valeurs fondatrices: la liberté et l'égalité en tension}

La vision bipolaire du système, proposée ici, est déjà dans les connotations et les hiérarchies constatables au sein du sacro-saint consensus fondateur, comme d'emblée suggéré. Le consensus n'étant rien d'autre qu'un compromis, mais un compromis sui generis, portant en la circonstance sur le fondamental; un compromis ample, voire général et accepté comme durable. Dans ces conditions, il suppose une certaine souplesse d'application, c'est-à-dire d'interprétation qui, en partie, revient au juge, mais, en partie aussi - au Président de la République, gardien de la constitution et du fonctionnement régulier des pouvoirs publiques, dans le respect de l'esprit de l'ensemble. On se souvient de la célèbre formule du Général de Gaulle sur la constitution comme un texte, une pratique et, surtout - "un esprit». Cette fonction arbitrale du président consiste, plus spécifiquement et avant tout à garantir l'équilibre entre nos deux paradigmes, libéral et démocratique, à travers les différentes normes juridiques et rapports des pouvoirs politiques.

Les valeurs "partagées», libérales et démocratiques, bien que cohabitant au sein du consensus fondateur du système, rentrent souvent en concurrence; dans la vie réelle comme dans les discours qui les approfondissent D'ailleurs, toute l'histoire de la démocratie libérale était sous-tendue par les problèmes de la frontière légitime entre les exigences de la liberté e celles de l'égalité. Souvenons-nous des débats législatifs sous la IV ${ }^{\text {ème }}$ République française. A chaque initiative législative significative de la "droite libérale - classique», quel qu'en soit l'expression partisane concrète, la "gauche démocratique - moderne» opposait le contre argument face au «libéralisme exacerbé», c'est à dire le retour à l'état de nature, "sauvage», assurant, en fait, le règne de la loi du plus fort et non pas du plus légitime. Et, à l'inverse, symétriquement, toute initiative d'ampleur de la "gauche démocratique- moderne» ${ }^{10}$ était dénoncée comme "égalitariste» et, à ce titre, «liberticide». Ainsi vont normalement les compromis démocratiques, entre les valeurs, comme entre les intérêts.

La liberté et l'égalité sont, en effet, les deux «désirs», pulsions ou exigences - profonds de toutes les sociétés, notamment modernes. Ce sont les deux piliers idéologiques de la civilisation "démo-libérale» chargés d'en sublimer les contradictions de façon pacifique et rationnelle. En France, c'est le compromis historique entre Montesquieu, le libéral et Rousseau, le démocrate, qui symbolise cette dialectique des valeurs fondatrices. Tous les pouvoirs y ont contribué, le constituant, le législatif, l'exécutif et, bien sûr, le judiciaire aussi. Tous ceux qui sont sensés appliquer les normes fondatrices, en respectant surtout l'esprit des normes, car les normes sont souvent "recyclés» dans leurs sens exégétiques et formels concrets, conformément aux changements induits par l'évolution du terrain social, alias politique, auquel le droit doit s'adapter. C'est la fonction du juge qui est le mieux à même d'opérer ce changement en souplesse, par ses "arrêts de principe» ou les "star décisions» issus des «virages jurisprudentiels». C'est ainsi que le juge participe activement aux mutations du système politique par la voie juridique, en évitant les «frais généraux» de

\footnotetext{
${ }^{10}$ Nous retrouvons ici le vocabulaire, bien consensuel, de l'époque.
} 
la violence révolutionnaire ou autre. Le poids politique substantiel du juge est ici aussi évident que légitime.

Les valeurs "communes» se retrouvent dans un noyau dur - minimum, qui est véritablement "commun» ou "partagé». Au-delà de ce noyau, dans l'enveloppe "molle», nos deux mouvances idéologiques se différencient, voire se séparent et peuvent rentrer en conflit, politique et juridique, sans pour autant remettre en cause le consensus systémique.

La liberté est une aspiration profonde de toute l'humanité, constatent les libéraux. C'est la priorité idéologique absolue. De leur ouvance. Bien entendu, elle ne peut prospérer dans l'espace social sans une certaine égalité; mais, en même temps, «trop d'égalité, tue la liberté», car, c'est alors l'égalitarisme. Mené au bout de sa logique, celui-ci supprime la liberté, sans pour autant construire une égalité réelle, comme l'expérience du socialisme marxiste nous l'a démontré, tout au long $d u X X^{\text {ème }}$ siècle. La liberté est donc un droit naturel, c'est-à-dire universel, fondé sur la Raison qui, elle - aussi, a un statut d'universalité. A ce titre elle est plus affirmée que débattue, puisqu'il s'agit d'un axiome fondateur.

L'idée de liberté a généré toute une série de droits et libertés, dont la dignité de la personne humaine, qui est à la pointe de l'actualité. Comment nier le rôle immense du juge dans l'interprétation de cette valeur, devenu principe et donnant lieu à des droits au respect dans les contextes et des situations différents. On touche là directement aux fondements idéologiques et intérêts politiques de nos deux mouvances.

Il en va de même avec les idées qui sous-tendent la valeur égalitaire. Pour la mouvance démocratique, l'égalité est le préalable à la liberté pour tous. Le contrat social, politique et démocratique pré-conditionne la bonne distribution et le bon usage de la liberté. Or, une fois de plus, c'est en matière politique seulement que l'on peut construire cette valeur à partir du principe ONE MAN - ONE VOTE. C'est ainsi que l'on retrouve le paradigme sociétal ou collectif, c'est-à-dire politique, consubstantiel à la démocratie. Et, tout comme la liberté, l'égalité a généré une filière de droits «acquis» par des luttes politiques, voire historiques, et désormais installées au sein des valeurs fondatrices, bien intériorisés.

\section{B. Le juge entre la légalité juridique et la légitimité politique}

La question concerne ici les problèmes d'interprétation de la conformité hiérarchique de la norme juridique dans le contexte politique à légitimité évolutive.

Au préalable, il nous semble utile, une nouvelle fois et après la mise en évidence de la tension entre les valeurs, de revoir le cadre et l'esprit du système, à travers sa complexité technologique, qui sous-tend et marque le travail du juge.

Il y a, en effet, dans notre système de l'Etat de droit, deux technologies organisationnelles et fonctionnelles, qui sont en étroite symbiose et qui concourent, toutes les deux, au bon fonctionnement du système: le droit et la politique, le juridique et le politique, avec leurs techniques, procédures et instances diverses, dont la norme juridique et le vote politique sont les piliers. Si le système a besoin des deux technologies (devenues en partie, valeurs, voire même idéologies...), pour exister et fonctionner, celles-ci jouent des rôles différents, 


\section{Slobodan MILACIC}

préférentiels, voire déterminants, selon qu'il s'agit des «causes» ou des «mouvances» plus spécifiquement libérales ou démocratiques.

Le vocable «technologie» peut éventuellement surprendre, car il s'agit, en effet, aussi des valeurs; des idéologies, comprises comme systèmes de valeurs. II y a des idéologies juridiques et politiques, générées par les valeurs libérales et démocratiques, aux services desquelles se trouvent le droit et la politique. Pour la circonstance, nous voulons seulement mettre en exergue le juridique et le politique comme techniques d'organisation, et de régulation des rapports des pouvoirs politiques. Plutôt que simples techniques, le juridique et le politique sont de véritables systèmes technologiques, impliquant des procédés, des procédures et des institutions fonctionnelles diverses, nécessaires à la production et la gouvernance des normes et des décisions politiques qui, en tant que tels, peuvent être mis au service des «causes» substantiellement très différentes. Aucun pouvoir politique, aucun Etat, ne peut se passer, de nos jours de la modernité complexe, de droit constitutionnel, ni de procédé électoral. Tous les Etats n'existent que par le droit, même si tous ne sont pas des Etats de droit, beaucoup s'en faut. Les Etats de droit avérés, comme les démocraties libérales crédibles sont encore très minoritaires dans le monde d'aujourd'hui, constitué de 200 Etats, environ. II y a, parmi eux, beaucoup «Etats voyous» ou de "pouvoirs pervers» dont les discours et les apparences symboliques exhibées, en général, sont à l'inverse de la réalité. Le discours juridique et politique a pour fonction, dans ce cas, de faire croire, de compenser verbalement, symboliquement ou psychologiquement, le déficit de légitimité du pouvoir ou du système, lui-même. C'est pourquoi nous avons postulé que le droit et le politique peuvent, pour les besoins de l'analyse, être considérés comme des technologies "détachables» des valeurs dont elles sont les instruments. C'est pourquoi, finalement nous avons insisté sur les aspects complémentaires et inévitables du lien entre le juridique et le politique; à la fois pour la bonne marche et la bonne compréhension de notre Etat de droit, démocratique et libéral. Le système de complémentarité et de concurrence, de synergie et de contradiction, implique l' "esprit» de la séparation des pouvoirs: celui de la surveillance mutuelle, accompagnant la collaboration. Tout comme en matière de valeurs, le juge se trouve en réalité (souvent dénigrée, occultée ou critiquée) en position de plus ou moins grand écart, entre le texte et le contexte. II nous semble donc théoriquement légitime d'avaliser la substance politique du travail du juge; de ne pas en faire une cause de "mauvaise conscience», au nom d'une vision par trop «puriste» ou juriste de l'Etat de droit, car il s'agit d'une notion composite, fusionnant le juridique (le Droit) et le politique (l'Etat).

Ainsi on peut lire notre État de droit démocratique et libéral ou, désormais, libéral et démocratique, comme constitué de deux pyramides en interférence. Pyramides emboîtées, mais structurées de façons différenciées, selon qu'il s'agit du «sous-système» libéral ou du "sous-système» démocratique. Évoquons en quelques mots les cohérences propres de nos deux filières - matrices, perceptibles à tos les principaux stades des processus et permettant une plus globale et systématique lecture du système.

Le libéralisme, qui a plutôt tendance à donner "le pouvoir du dernier mot» au juge, considéré comme gardien, voire garant de la norme, se fonde sur le Droit naturel. Celui-ci est d'abord et avant tout celui de l'Homme, qui deviendra, avec la valeur démocratique, 
alias égalitaire, celui du Citoyen. II reste que pour le libéral le postulat fondateur affirme que c'est la norme, la loi ou la constitution, qui transcrit le droit naturel et le juge qui le garantit. Ainsi s'énonce la construction de l'ordre libéral: l'idéologie de la liberté, naturelle ou consubstantielle à l'homme, son affirmation par la norme juridique et sa garantie par le juge. Tout «naturellement» alors on débouche sur la conclusion que «le pouvoir du dernier mot» appartient au droit, c'est-à-dire au juge, qui dit le droit lorsque le problème se pose. Le pouvoir politique, tout comme le citoyen, doivent s'y soumettre. Le politique y est perçu comme le garant de l'ordre public, mais celui-ci est envisagé a minima, au profit de l'ordre privé, «naturel» et concurrentiel. Ce qui intéresse cette vision des choses c'est la virtualité, la possibilité ou la «chance» de pouvoir jouir de la liberté; pas l'usage réel qu'on en fait, qui dépend du collectif, qui, lui, intéresse plus spécifiquement la vision démocratique, c'est-à-dire politique des choses.

Le démocratisme, pour employer une sémantique similaire, bien qu'imparfaite, privilégie le politique, c'est-à-dire le collectif et le vote comme expression directe du Souverain - peuple. L'égalité est ainsi obtenue par les luttes politiques, avant d'être affirmée par les normes juridiques. Celles-ci évoluent nécessairement avec la société elle-même et seul le peuple, par le suffrage universel (bien entendu - égal) peut être le censeur ou le constituant, "en dernière instance». La vision démocratique s'intéresse à l'usage politique de la liberté; aux choix des institutions et des politiques publiques qui déterminent notre vie en société et pèsent sur nos destins collectifs et individuels. La liberté est, en effet, la condition et la finalité de toutes les autres légitimités dérivées, l'alpha et l'oméga de notre système de valeurs. Mais, entre les deux pôles référentiels, entre la première et la dernière lettre de l'alphabet, il y a la vie, qui s'écrit politiquement, en termes de délibérations et de décisions - collectives. Ainsi, dans les démocraties libérales, si le "pouvoir du dernier mot» en droit appartient normalement au juge, qui l'exerce de façon "ordinaire» ou routinière, en même temps, la loi ou la norme sont au départ, il ne faut pas l'oublier, des actes politiques, issus des votes qui leur confèrent leur qualité substantielle, leur «identité», c'est-à-dire le «sens», en fonction duquel on délimite la "portée». Par ailleurs et symétriquement, les normes juridiques sont, en démocratie et par hypothèse, des compromis politiques. Pour leur donner un sens, pour les interpréter, le juge doit faire une analyse politique du compromis, qui est la substance de toute norme juridique. Nous sommes encore dans le lien indissoluble entre le juridique et le politique. Mais le cercle vertueux des interdépendances ne s'arrête pas là, il ne s'arrête pratiquement jamais dans l'Etat de droit: pour qu'un vote soit légitime, encore faut-il qu'il respecte une procédure ou des conditions juridiques de procédure préétablies. Le juridique et le politique fonctionnent de ce point de vue comme un double circuit, de complémentarité et de sécurité.

Il y a donc, en démocratie libérale, un cercle vertueux des interdépendances qui donne une certaine souplesse au jeu du "pouvoir au dernier mot» qui se passe entre le juridique et le politique; entre les pouvoirs politiques et le pouvoir judiciaire de façons complexe et variables, selon les contextes légitimant et les habitus technologiques.

Le juge est le garant des droits et des libertés constitutionnelles, mais son travail d'interprétation implique l'évaluation de la légitimité dans le contexte. Derrière chaque 


\section{Slobodan MILACIC}

évaluation de la légalité, alias constitutionnalité, se cache une appréciation de la situation contextuelle, en termes de légitimités concurrentes. Car, l'évaluation du rapport de forces en démocratie se fait en termes de légitimités. C'est un travail politique qui implique le recours au terrain, aux croyances de l'opinion et pas seulement aux concepts juridiques et à leur hiérarchie. Le Président de la République, pouvoir exécutif, politique par excellence, est aussi un arbitre, garant des institutions. II exerce cette fonction, voire cette mission, en évaluant l'équilibre politique des pouvoirs; conformément aux dispositions et à l'esprit de la constitution, cependant. A cet égard il est, en quelque sorte en position de symétrie inversée par rapport à celle du juge, qui est une instance primordialement juridique, avant d'être politique.

Finalement, la constitution a deux gardiens: le juge constitutionnel et le Président de la République. L'un, principalement juridique, mais interférant dans le politique. L'autre, plutôt politique, mais dans le respect de la constitution. L'un contrôle la conformité des normes par rapport à la constitution, en faisant son évaluation en fonction des exigences contextuelles de légitimité; en équilibrant le jeu des exigences de la liberté et ceux de l'égalité, «en dernière instance». L'autre, en arbitrant le rapport de forces politiques légitimes, mais dans l' "esprit de la constitution», en termes d'équilibre des pouvoirs, par exemple. Mais le Président de la République connaît des limites dans l'exercice de cette fonction, car, tout comme le juge, il n'est pas Le Souverain. C'est un pouvoir constitué, pas constituant. Finalement, le vrai dernier mot appartient au constituant, en matière constitutionnelle.

On constate souvent, dans les systèmes néolibéraux notamment, des hésitations sur le "pouvoir du dernier mot», entre la légalité et la légitimité, lorsqu'elles sont en concurrence ou en contradiction. La légalité est juridique, alors que la légitimité est politique. Or, «en dernière instance», c'est toujours la question de légitimité qui se pose. Mais, même dans ce cas de figure, la question de la crédibilité ou de la légitimité propre du droit ou du vote, en tant que techniques, interfèrent et pèsent en tant que telles, comme valeurs spécifiques; indépendamment ou en combinaison avec la substance idéologique ou idéelle, sensées être décisives dans l'appréciation de la légitimité. Parfois c'est l'instrument qui porte l'artisan!

\section{En guise de conclusion}

Le juge est devenu une instance primordiale dans le système néolibéral de l'Etat de droit, mais, en même temps, la place du juge par rapports aux autres pouvoirs et par rapport au politique, en général, n'est pas suffisamment élucidée. II ne suffit pas d'affirmer qu'il a le «droit au dernier mot», puisqu'il se prononce «au nom du peuple français». Dans le modèle général de la démocratie pluraliste, qui nous inspire en fondant nos régimes, le juge n'est pas Robinson Crusoé ...l'homme seul sur son île déserte, décidant librement et souverainement, en toute virtualité du droit positif! II n'est qu'une pièce d'un système politique et humain complexe; instance primordiale, comme telle, mais pas forcément la 
plus décisive pour dire le «dernier mot»; mais pas «nulle», non plus, comme le prétendait Montesquieu, pour des raisons historiques et conjoncturelles spéciales, pour ne pas die spécieuses... La description du nouveau statut du juge dans le système de gouvernement, sa nouvelle popularité et même sa légitimité renforcée, ses pouvoirs élargis, en droit et en fait, tout cela nous permet de mieux connaître la mutation néolibérale de l'institution. Mais pour mieux comprendre le «nouveau juge» il faut le situer dans le système où il a sa place et son rôle - spécifiques, qu'il partage avec les autres pouvoirs - concurrents, dans l' "esprit du système», qui relativise le rôle de chacun. Si le juge a le "pouvoir du dernier mot» juridique, dans le cadre du fonctionnement normal, c'est-à-dire "ordinaire» ou "régulier» du système, dès les premiers «blocages», "crises» ou "circonstances exceptionnelles» en tous genres, c'est l'arbitrage politique qui prend la relève. Le législateur ou le constituant, par la voie des "validations législatives», parfois à la limite de la perversité (cf. la pratique sous la présidence V. Giscard d'Estaing) par la voie des réformes législatives (ou de "validations législatives», plus que douteuses, comme sous la présidence V. Giscard d' Estaing) et des révisions constitutionnelles, plus ou moins radicales, imposent au juge la nouvelle norme; en l'obligeant à abandonner sa jurisprudence, jusque-là «en vigueur».

On évoque régulièrement $l$ " «indépendance du juge» comme argument primordial de sa légitimité, en pensant traditionnellement à l’Exécutif ou au Législatif, c'est-à-dire au politique, alors qu'aujourd'hui on peut remarquer une certaine ouverture, voire une certaine disponibilité par rapport à l'opinion publique, comme si celle-ci devait exprimer plus directement et de façon plus actuelle la volonté du peuple souverain? On perçoit moins fort, en tout cas, le problème de l'indépendance du juge vis à vis de l'opinion publique, alors que les analyses critiques de l'opinion publique par rapport à l'exigence démocratique ne manquent pas d'arguments, pourtant! Cette «justice d'opinion», du moins en partie, réminiscence perverse de la «justice populaire» d'autrefois, révèle une percée incontrôlée du juge dans le politique contextuel, confondant malheureusement, en la circonstance, la popularité et la légitimité!

Dans le système complexe de l'Etat de droit libéral et démocratique qui nous gouverne, le juridique et le politique sont unis devant les hommes par le «contrat social»; évidemment, "pour le meilleur et pour le moins bon». On ne peut pas séparer, de façon absolue, le juridique du politique, pas plus qu'on ne peut séparer la liberté de l'égalité. Et pourtant, pour cette part du politique qui intervient inévitablement dans le juridictionnel, si limité soit-elle, on se refuse à lui reconnaître une certaine légitimité (ou même une certaine réalité!) et, par conséquent, de l'analyser comme telle. Et pourtant, «chassez le politique, il revient au galop», car il est dans la nature profonde du système de la démocratie libérale de jouer, à travers les combinatoires diverses, du juridique et du politique. Pour des raisons techniques, matérielles ou technologiques, propres à tout pouvoir étatique moderne, mais aussi, pour l'Etat de droit qui nous intéresse et qui doit gérer les compromis entre les deux filières fondatrices avec leurs techniques préférentielles respectives. Les libéraux et les démocrates ont besoin du droit et de la politique, seulement dans les combinatoires différentes, souvent. Montesquieu l'avait déjà compris: aucun pouvoir ne peut être utilement "séparé» de l'autre de façon rigide ou absolue. L'autonomie n'est pas 


\section{Slobodan MILACIC}

l'indépendance! Dans une Cité libérale qui le préoccupait, il y a nécessairement un minimum d' «interdépendance organique» et de "collaboration fonctionnelle», disait-il, en substance, dans son fameux chapitre sur "La constitution d'Angleterre». Ce qui est vrai pour les "pouvoirs», en tant que tels, l'est aussi pour les paradigmes technologiques qui en sont la logistique: le juridique et le politique, sous toutes leurs formes respectives. Si la séparation "rigide » des pouvoirs n'est nulle part possible, la séparation rigide du juridique et du politique n'est pas pertinente pour l'Etat de droit, non plus. C'est ce que nous voudrions faire valoir ici, à propos du juge et de l'Etat de droit, "revus et corrigés» par le néolibéralisme. Le juge est le gardien des libertés juridiques et de la démocratie politique et, à ce titre, il peut et doit accomplir une analyse de l'objet politique et, finalement, dans sa décision, impliquer un choix politique ou idéologique. Il vaut mieux le savoir et le contrôler que l'ignorer ou feindre de l'ignorer...

On a trop souvent tendance à confondre le fameux et proscrit «gouvernement des juges», comme parfaitement illégitime dans un 'Etat de droit démocratique, même si celui-ci n'impliquait qu'une participation sui generis et limité du juge au gouvernement de la Cité. Après tout il s'agit d'un "pouvoir» qui, si spécifique soit-il, participe du génie profond du système, en emboîtant aussi bien les valeurs que des techniques fondatrices. En tant que gardien des libertés individuelles et garant de la démocratie politique le juge est nécessairement impliqué dans les idées et les procédés politiques. D'une certaine façon et jusqu'à un certain degré, bien évidemment, mais même cela, le monde positiviste n'est pas encore prêt à le reconnaître. 